\title{
Somatosensory processing of the tongue in humans
}

\author{
Kiwako Sakamoto ${ }^{1,2}$, Hiroki Nakata ${ }^{1,3}$, Masato Yumoto $^{2}$ and Ryusuke Kakigi ${ }^{1 *}$ \\ Department of Integrative Physiology, National Institute for Physiological Sciences, Okazaki, Japan \\ 2 Department of Clinical Laboratory Medicine, Graduate School of Medicine, The University of Tokyo Hospital, Tokyo, Japan \\ ${ }^{3}$ Faculty of Sport Sciences, Waseda University, Tokorozawa, Saitama, Japan
}

\section{Edited by:}

Jean-Pierre Montani, University of

Fribourg, Switzerland

\section{Reviewed by:}

Elizabeth Disbrow, University of

California at Davis, USA

Nobukazu Nakasato, Tohoku University, Japan

\section{${ }^{*}$ Correspondence:}

Ryusuke Kakigi, Department of

Integrative Physiology, National

Institute for Physiological Sciences,

Okazaki, 444-8585, Japan.

e-mail: kakigi@nips.ac.jp
We review research on somatosensory (tactile) processing of the tongue based on data obtained using non-invasive neurophysiological and neuroimaging methods. Technical difficulties in stimulating the tongue, due to the noise elicited by the stimulator, the fixation of the stimulator, and the vomiting reflex, have necessitated the development of specialized devices. In this article, we show the brain activity relating to somatosensory processing of the tongue evoked by such devices. More recently, the postero-lateral part of the tongue has been stimulated, and the brain response compared with that on stimulation of the antero-lateral part of the tongue. It is likely that a difference existed in somatosensory processing of the tongue, particularly around primary somatosensory cortex, Brodmann area 40, and the anterior cingulate cortex.

Keywords: SI, SII, fMRI, MEG, tongue, oral, maxillofacial

\section{INTRODUCTION}

The tongue is essential for daily life. The tongue is an epithelial sac filled with muscles and connective tissue; these muscles can be controlled willfully and are generally referred to as skeletal muscles or voluntary striated muscles, which are divided into intrinsic and extrinsic muscles (Brand and Isselhard, 2003). In addition, the tongue has various functions: preservation of the position of the teeth and expression of feelings, speech, swallowing, and mastication. However, there has been relatively few neuroimaging and neurophysiological studies focusing on the functions of the tongue. There are several problems with studying somatosensory (tactile) processing of the tongue in humans. The first problem is the choice of a stimulator that can stimulate the tongue while the subject is under a scanning coil or electrodes without causing noise or technical problems. The second problem is that it is extremely difficult to fix the stimulator on the tongue stably, since the tongue itself is made of soft tissue and is convex in shape. The third problem is that tactile stimulation in this area frequently triggers the vomiting reflex. Taking these problems into consideration, ingenuity is required to record the brain activity associated with somatosensory processing of the tongue, and some researchers have developed devices, which are introduced in later sections, to solve these problems.

Recently, several non-invasive recording methods have been used to measure human brain activity. Among these are methods based on neurophysiology, including electroencephalography (EEG), magnetoencephalography (MEG), and methods based on neuroimaging, including functional magnetic resonance imaging (fMRI) and positron emission tomography (PET). EEG is indispensable for examining the neural activities in the human brain and offers a high temporal resolution on the order of milliseconds. EEG technology captures fluctuations in the electrical voltage of the brain through electrodes placed on the scalp in accordance with the standardized guidelines of the International 10-20 system
(Jasper, 1958). EEG data also represent changes in the potential differences between different points on the human scalp and the electric field potentials that arise from excitatory and inhibitory postsynaptic potentials. MEG offers several theoretical advantages over EEG in localizing cortical sources (brain dipoles) because the magnetic fields recorded on the scalp are less affected by volume currents and anatomical homogeneity. MEG also permits the spatial and temporal localization of excited cortical areas on the order of millimeters and milliseconds (reviewed in Hari et al., 2000; Kakigi et al., 2000). fMRI, which measures the blood oxygenation leveldependent (BOLD) signal, has been used not only as a tool for mapping brain activity but also as a means of studying the dynamics of neural networks by tracking fMRI response characteristics across various spatial and temporal scales (Logothetis et al., 2001). PET has been used to measure regional cerebral blood flow ( $\mathrm{rCBF}$ ) with the intravenous radioactively labeled water $\left(\mathrm{H}_{2}{ }^{15} \mathrm{O}\right)$ bolus technique (Decety et al., 1994). These recording methods have been used to clarify somatosensory processing of the tongue in humans.

In this review article, we examine recent non-invasive research showing the brain activity for somatosensory processing related to the tongue. To our knowledge, no systematic review of the scientific literature on somatosensory processing of the tongue is available despite much research. We argue that combining and reviewing non-invasive research will provide new insights because neurophysiological and neuroimaging methods have limitations when it comes to brain activity. We focus on three broad areas; primary somatosensory cortex (SI), secondary somatosensory cortex (SII), and neuroimaging data.

\section{RESPONSES FROM PRIMARY SOMATOSENSORY CORTEX}

Neurophysiological studies using EEG and MEG have helped to clarify the temporal dynamics of the tongue SI (Ishiko et al., 1980; Altenmüller et al., 1990; Karhu et al., 1991; Nakamura et al., 1998; Maloney et al., 2000; Disbrow et al., 2003; Nakahara et al., 2004). The 
evaluation of time-locked EEG and MEG following somatosensory stimulation (i.e., somatosensory-evoked potentials, SEPs; somatosensory-evoked magnetic fields, SEFs) constitutes one of the most useful methods for investigating the human somatosensory system. These studies showed SI activity in the postcentral gyrus, which is consistent with the homunculus reported by Penfield and Boldrey (1937).

One major problem with SEPs and SEFs is that the peak latency of the primary response has not been consistent. For instance, there is general agreement that SI responds after about $20 \mathrm{~ms}$ to electrical stimulation of the median nerve or finger (Hari et al., 1993; Mauguière et al., 1997; Kakigi et al., 2000). However, the response time to stimulation of the tongue ranges widely from 10 to $55 \mathrm{~ms}$ (Table 1). In addition, some studies reported activity in SI contralateral to the stimulation (Nakamura et al., 1998; Maloney et al., 2000; Nakahara et al., 2004), whereas others found activity in both SIs (Ishiko et al., 1980; Altenmüller et al., 1990; Karhu et al., 1991; Disbrow et al., 2003; Maezawa et al., 2008; Sakamoto et al., 2008a; Tamura et al., 2008).

As mentioned in the Introduction, there might be several reasons why consistent results have not been recorded for tongue somatosensory stimulation. Thus, researchers have made new devices to measure stable brain responses in SI.

Ishiko et al. (1980), who were the first to investigate SEPs elicited by stimulating the tongue, applied mechanical stimulation to the right anterior region of the tongue as it protruded slightly from the mouth. The striking surface of the probe was square and flat with an area of $1 \mathrm{~mm}^{2}$. Its striking strength, in terms of tension developed while tapping the tongue, was found to be $10 \mathrm{~g}$.

Altenmüller et al. (1990) used a modified EEG ear clip electrode ( $5 \mathrm{~mm}$ in diameter) located on either side of the tip of the tongue with the cathode on the upper and the anode on the lower side and vice versa. To avoid synchronous electrical stimulation of the oral mucosa and the lips, the outer side of the electrode was electrically isolated. During stimulation, the tongue was held relaxed inside a slightly opened mouth.

Karhu et al. (1991) used a hand-made clip electrode consisting of two Ag plates (diameter $=5 \mathrm{~mm}$ ), and delivered current to the anterior left side of the tongue. The outside of the electrode was

Table 1 |The peak latency of the primary response after tongue stimulation in previous studies (EEG and MEG).

\begin{tabular}{llll}
\hline Reference & Recording & $\begin{array}{l}\text { Latency of primary } \\
\text { response (ms) }\end{array}$ & $\begin{array}{l}\text { Recorded } \\
\text { site }\end{array}$ \\
\hline Ishiko et al. (1980) & EEG & 13 & Contr, Ipsil \\
Altenmuller et al. (1990) & EEG & 21 & Contr, Ipsil \\
Karhu et al. (1991) & MEG & 55 & Contr, Ipsil \\
Nakamura et al. (1998) & MEG & 36 & Contr \\
Maloney et al. (2000) & EEG & 13 & Contr \\
Disbrow et al. (2003) & MEG & 10 & Contr, Ipsil \\
Nakahara et al. (2004) & MEG & 55 & Contr \\
Tamura et al. (2008) & MEG & 14 & Contr, Ipsil \\
Maezawa et al. (2008) & MEG & 23 & Contr, Ipsil \\
Sakamoto et al. (2008) & MEG & 19 & Contr, Ipsil \\
\hline
\end{tabular}

Contr, contralateral hemisphere to the stimulation; Ipsil, ipsilateral hemisphere to the stimulation. insulated to avoid synchronous stimulation of the oral mucosa. The tongue was held relaxed inside the mouth which was slightly opened to allow leads to come out between the upper and lower teeth.

Nakamura et al. (1998) used an air-puff-derived tactile stimulator, which provides a light, superficial pressure stimulus to the skin. The area of contact by the circular rubber bladder was $1 \mathrm{~cm}$ in diameter, and the intensity of the mechanical stimulation was $40 \mathrm{~g} / \mathrm{cm}^{2}$. The rise time was $20 \mathrm{~ms}$ as measured at $10-90 \%$ of the intensity.

Maloney et al. (2000) used pairs of thin, stainless steel disk electrodes on modified mandibular or maxillary acrylic splints, similar to orthodontic retainers. The Mandibular splint electrodes were oriented to make contact with the under surface of the tongue along the course of the right and left lingual nerves and the maxillary splint electrodes were oriented to make contact with the hard palate bilaterally along the course of the palatine nerves.

In the MEG study of Disbrow et al. (2003), stimuli consisting of pneumatically driven mechanical taps were applied to the tongue with a balloon diaphragm $1 \mathrm{~cm}$ in diameter. The diaphragm was placed as far from the midline as possible, near the edge of the tongue.

Nakahara et al. (2004) used a clip electrode with a 5-mm interelectrode distance, which was attached to the tongue mucosa 5-10 $\mathrm{mm}$ from the lingual edge.

Maezawa et al. (2008) used a pair of pin electrodes. The interelectrode distance was $3 \mathrm{~mm}$. Three points on the dorsum of the tongue were stimulated in the following order: (1) the right side $(2 \mathrm{~cm}$ from the tip of the tongue, $1 \mathrm{~cm}$ from the edge), (2) the left side (symmetric to the right side), and (3) the midline ( $1 \mathrm{~cm}$ from the tip of the tongue). Before recordings were made, the three points were marked with crystal violet. A pair of epoxy resin-coated platinum pin electrodes ( $0.4 \mathrm{~mm}$ in diameter) was used for stimulation.

In the MEG study of Tamura et al. (2008), the device used for stimulation was modified from Braille cells for the visually impaired. It consisted of a piezoelectric element and stimulus pins pushed out $(0.7 \mathrm{~mm} / 0.4 \mathrm{~ms})$ by application of a direct current to the piezoelectric element to produce tactile stimulation of the area targeted. Eight stimulus pins were aligned, with a gap of $2.4 \mathrm{~mm}$ between each. The stimulation was $0.18 \mathrm{~N}$ in force.

More recently, we fabricated an intraoral device for each individual using hydrophilic vinyl silicone impression material (EXAFAST/ Putty Type, GC, Japan), and recorded SEFs (Sakamoto et al., 2008a) (Figure 1A). The subject bit bilaterally into the EXAFAST, which was mixed uniformly and formed into two blocks. The jaws of the subjects were positioned based on centric occlusion and opened about $8 \mathrm{~mm}$ between the upper and lower teeth to make a small space that was important to build the electrode for stimulating the tongue. These blocks were used to create a space from the right to left central incisor teeth to allow relaxation of the tongue, and to keep the jaw in a mandibular rest position. Then, we made four holes, which passed from the buccal to lingual side of the device, positioned on the lingual cusp of the canine teeth and the distallingual cusp of the second molar teeth of the mandible bilaterally. Next, we made a concentric bipolar electrode, which could be set in each hole (Figure 1B). This electrode has a cathode consisting of a silver wire (1.0 mm in diameter) and a cylindrical anode consisting of stainless steel $(3.5 \mathrm{~mm}$ in diameter and a gap between the anode and cathode of about $0.9 \mathrm{~mm}$ ). To extend the line, the 
A
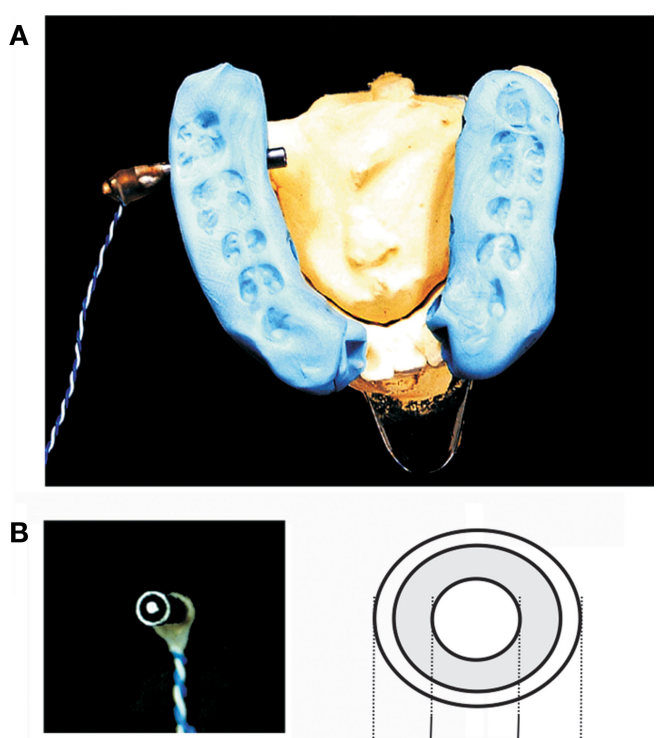

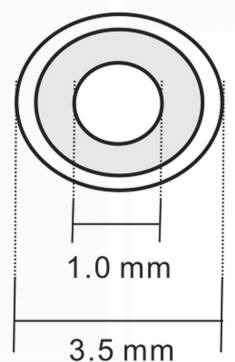

$3.5 \mathrm{~mm}$

FIGURE 1 | (A) Device used to stimulate the tongue. The electrode's position was determined on the right and left antero-lateral margins and the postero-lateral margins of the tongue around foliate papillae by using the device. (B) The surface structure of the concentrated bipolar electrode used to stimulate the tongue. Adopted from Sakamoto et al. (2008a).

silver wire $(0.2 \mathrm{~mm}$ in diameter) was fixed with tin solder to the cathode and anode. The cathode and anode were fixed by polymethylmethacrylate, including the points soldered. This electrode was easily attached and detached, but very stable during the experiments (Figure 1B). SEFs were recorded by using these devices, and compared following stimulation of the right and left postero-lateral parts of the tongue, and right and left antero-lateral parts. The primary component was recorded about $19 \mathrm{~ms}$ post-stimulation. Six components, termed $1 \mathrm{M}, 2 \mathrm{M}, 3 \mathrm{M}, 4 \mathrm{M}, 5 \mathrm{M}$, and $6 \mathrm{M}$, respectively, were found within $130 \mathrm{~ms}$ of the stimulation (Figure 2). These activities were detected in hemispheres both contralateral and ipsilateral to the stimulation, and estimated to be located around the tongue SI (Figure 3). The latency of the contralateral hemisphere was significantly shorter than that of the ipsilateral hemisphere for all components, independent of the area stimulated. There was no significant difference of coordinates in the somatotopic representation between antero-lateral and postero-lateral parts of the tongue. Some anatomical studies reported that the somatotopic representation in the tongue SI differed between anterior and posterior parts (Manger et al., 1995, 1996; Jain et al., 2001). This discrepancy between anatomical studies and our finding should be related to the limitation of spatial resolution in MEG.

Taking previous studies of SEPs and SEFs into consideration, the peak latency of the primary response would be obtained within $20 \mathrm{~ms}$ after stimulating the tongue, and in some reports, true primary cortical response might be missing, because of the difference of stimulating device. In addition, the orientation of equivalent

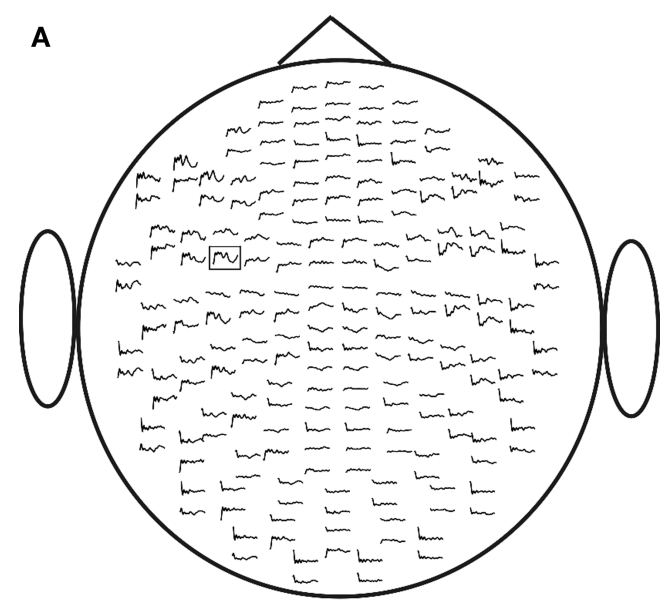

B

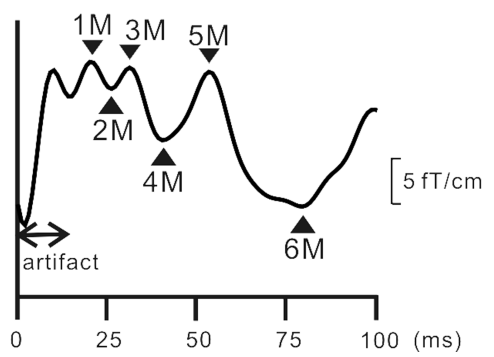

FIGURE 2 | Grand averaged MEG signals for the right anterior (RA) stimulation of the tongue. (A) The SEF waveforms over 204 planar coils from the top of the head. (B) An enlarged waveform recorded in the left centrotemporal areas, which was framed by a square in $(\mathbf{A})$. Adopted from Sakamoto et al. (2008a).

current dipole (ECD), which was estimated in MEG studies, is also listed in Table 1, and some studies showed the inferior-posterior direction (Karhu et al., 1991; Nakamura et al., 1998; Nakahara et al., 2004; Maezawa et al., 2008). All these studies indicated the primary response at more than $20 \mathrm{~ms}$. On the other hand, Tamura et al. (2008) and Sakamoto et al. (2008a) showed the primary response within $20 \mathrm{~ms}$, and the superior-anterior or inferior-anterior directions (Sakamoto et al., 2008a; Tamura et al., 2008). After stimulating median nerve, the primary response was recorded at about $20 \mathrm{~ms}$, and the ECD demonstrated anterior direction (Karhu et al., 1991; Wasaka et al., 2003; Huttunen et al., 2006). Several previous studies also have provided evidence that the primary response obtained within $20 \mathrm{~ms}$ indicated anterior direction after stimulating face and oral regions, such as buccal (Tamura et al., 2008), lip (Tamura et al., 2008), and hard palatine (Bessho et al., 2007). Therefore, we inferred that the true primary response, which was recorded within $20 \mathrm{~ms}$ after stimulation of the tongue, should show the anterior direction in ECD, and the second and sequential responses show the posterior direction.

As a characteristic of somatosensory processing of the tongue, neural activation was found in contralateral and ipsilateral hemispheres to the stimulation. Seven in 10 previous studies reported the bilateral activities (Table 1). For example, MEG studies showed that the difference of the latency between the contralateral and ipsilateral hemisphere was about $1 \mathrm{~ms}$ for P40m in Maezawa et al. 


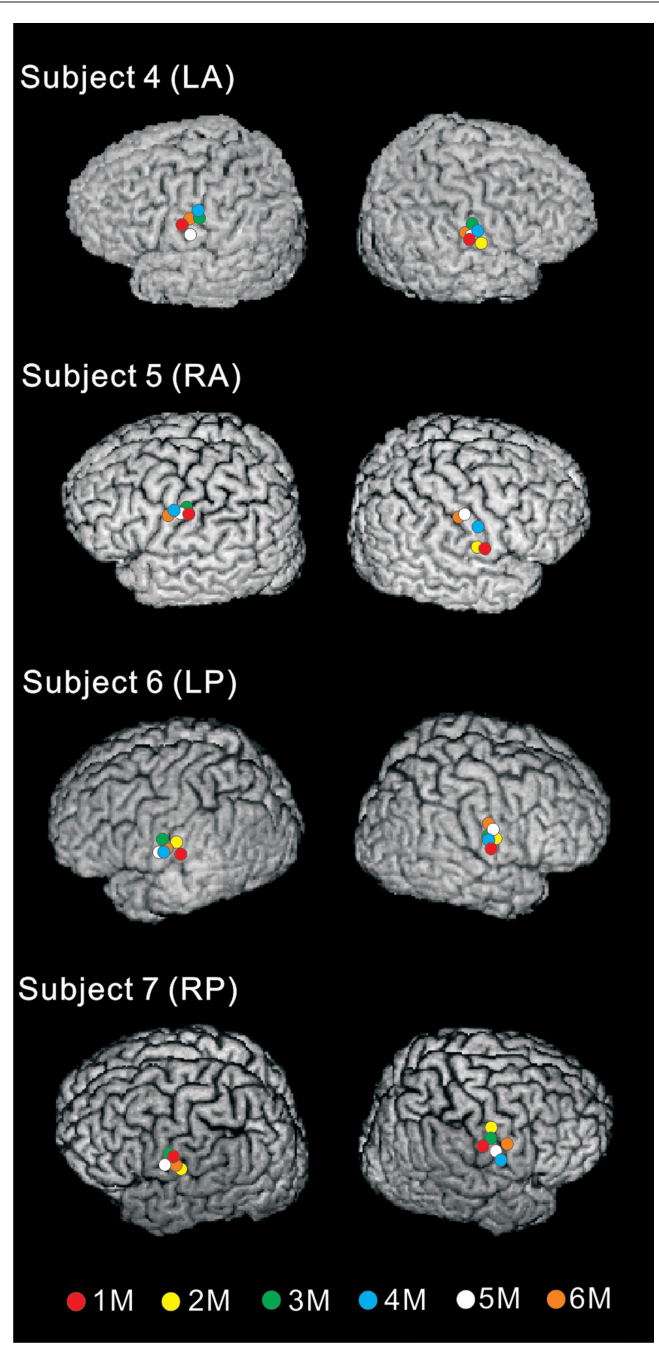

FIGURE 3 | Equivalent current dipoles (ECDs) on 3D images of four representative subjects. These ECDs were estimated to lie around the posterior wall of the central sulcus, corresponding to the tongue SI. Red, yellow, green, blue, white, and orange circles indicate the ECDs of $1 \mathrm{M}, 2 \mathrm{M}$ $3 \mathrm{M}, 4 \mathrm{M}, 5 \mathrm{M}$, and 6M, respectively. Adopted from Sakamoto et al. (2008a).

(2008), $1 \mathrm{~ms}$ for $1 \mathrm{M}$ (14 ms) in Tamura et al. (2008), $1 \mathrm{~ms}$ for $1 \mathrm{M}$ (19 ms) component in Sakamoto et al. (2008a). Disbrow et al. (2003) reported that the second peak at $30 \mathrm{~ms}$ was the largest and most consistent response in the ipsilateral hemisphere and was used for additional analysis. The 10- and 55-ms peaks were reduced or absent in the ipsilateral responses, and there were no significant differences for contralateral vs. ipsilateral latency of activation (range of means $=31.7-39.5 \mathrm{~ms}$ ). On the other hand, in previous MEG studies using median nerve stimulation, the cortical response from SI was rarely recorded, and the characteristic of somatosensory processing of the hand differed from that of the tongue. Korvenoja et al. (1995) reported dipolar field distributions over the ipsilateral SI in 5 of the 10 subjects after median nerve stimulation, and three subjects showed the response at 140-150 ms after stimulation, one at 80-100 ms, and one at 290-300 ms. In the somatosensory processing of the tongue, we considered that ipsilateral projection from contralateral hemisphere was irrelevant to the corpus callosum, and there was ipsilateral direct projection via an uncrossed ascending pathway from the trigemino-thalamic tract to SI.

\section{RESPONSES FROM SECONDARY SOMATOSENSORY CORTEX}

Secondary somatosensory cortex is located in the parietal operculum in the upper bank of the Sylvian fissure. Its existence has been known since early cortical stimulation studies during epileptic surgery (Schnitzler and Ploner, 2000).

In addition to the tongue SI, the neural activities and the somatotopic representation of the tongue SII should be clarified. Compared with SI, SII has been speculated to serve a higher level of cognitive function in somatosensory processing, such as attention, decisionmaking, object recognition, and the integration of nociceptive and non-nociceptive inputs (Mima et al., 1998; Steinmetz et al., 2000; Romo et al., 2002; Torquati et al., 2003; Inui et al., 2004; Nakata et al., 2004; Wasaka et al., 2005). The notion that SII is higher than SI in hierarchy was proposed on the basis of their anatomical relationships: SI sends projections to SII, while SII projects back to the superficial layers of SI (see a review, Iwamura, 1998). Studies in monkeys also showed that a unilateral lesion of SII impaired tasks of tactile learning and retention (Ridley and Ettlinger, 1976, 1978; Garcha and Ettlinger, 1978), and patients with lesions of SII had tactile agnosia (Caselli, 1993). Thus, investigating the neuronal activities of the tongue SII is important to understand the characteristics of somatosensory processing of the tongue, based on several aspects such as source location, source orientation, functional features and comparison with other somatic SII responses (Schnitzler et al., 1999).

To our knowledge, two studies have examined the neural activities of the tongue SII using MEG. In one previous study, Disbrow et al. (2003) showed the responses of the tongue SII after stimulating the edge of the tongue. They also recorded the lip SII, but did not compare the latency, location, and strength of brain responses directly and statistically between them.

In our study, we used individual intraoral devices and a concentric bipolar electrode, and recorded SEFs after stimulating four body sites, the left antero (LA) and postero (LP) lateral margins of the tongue, left median nerve at the wrist (hand), and left tibial nerve at the ankle (foot) (Sakamoto et al., 2008b). Neural activities were recorded from bilateral SII in both hemispheres after the four sites were stimulated. The activity of the tongue SII was recorded 80-110 ms after the stimulation (Figure 4). The tongue SII for LA and LP was located close to the hand SII and significantly more anterior than the foot SII, and there was no significant difference in the location of dipoles between the LA and LP areas of the tongue SII (Figure 5). The tongue SII was located very close to the hand SII, and significantly apart from the foot SII, showing a more anterior location than the foot SII (Figure 6). These findings concerning the locations of ECDs for SII imply the existence of an antero-posterior and infero-superior arrangement of the body surface in SII, which was consistent with some previous studies using MEG (Maeda et al., 1999; Nguyen et al., 2005), fMRI (Del Gratta et al., 2000, 2002; Disbrow et al., 2000; Ruben et al., 2001), and monkeys (Burton and Carlson, 1986; Krubitzer et al., 1986; Cusick et al., 1989). A rough somatotopic representation exists within SII regions, that is, the face, upper limb, and lower limb are located from the lateral to medial region in this order. The mean peak latencies of the tongue 


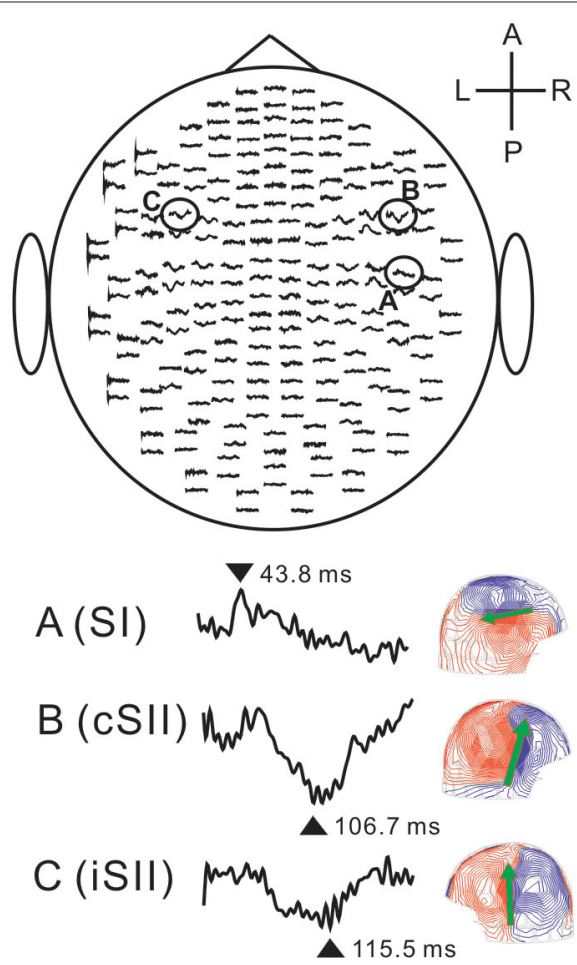

FIGURE 4 | MEG signals for the left anterior (LA) stimulation of the tongue in a representative subject. The upper figure shows the SEF waveforms over 204 planar coils from the top of the head. Lower-left figures indicate enlarged waveforms recorded in three areas, $A, B$, and C. Lower-right figures show each magnetic field pattern at 43.8, 106.7, and $115.5 \mathrm{~ms}$, respectively. The patterns are shown on the sensor array viewed from right (SI and CSII) and left (iSII). The arrows indicate the orientation of the ECD. Comparison between $\mathrm{A}$ (SI) and B (CSII) revealed clearly different field distributions and that the ECD of $A(S I)$ was directed posteriorly, while the ECDs of $B$ (cSII) were directed superiorly. $L$, left; $R$, right; $A$, anterior; $P$, posterior; SI, primary somatosensory cortex; cSII, secondary somatosensory cortex contralateral to the stimulation; iSII, secondary somatosensory cortex ipsilateral to the stimulation. Adopted from Sakamoto et al. (2008b).

SII for LA and LP were significantly shorter in the hemisphere contralateral to the stimulation than the ipsilateral hemisphere. These findings indicated that the tongue areas occupied a small region in SII with insufficient spatial separation to differentiate anterior from posterior areas even using MEG which has a higher spatial resolution than EEG.

\section{NEUROIMAGING STUDIES}

Recently, several neuroimaging studies using fMRI and PET have reported human brain activities evoked by somatosensory stimulation of the tongue, to clarify its somatotopic representation (Sakai et al., 1995; Pardo et al., 1997; Miyamoto et al., 2006; Minato et al., 2009). As compared with research on somatosensory processing of the hand and foot, a typical electrical stimulation can not be performed during fMRI recordings, when the tongue area is stimulated, because a magnetic body must not be put into the MR gantry. Thus, some ingenuity is needed to stimulate the tongue.

In an fMRI study, Sakai et al. (1995) stimulated the tip of the tongue from the medial side to the right side (2-cm long) using a cotton swab (stick diameter, $2 \mathrm{~mm}$ ) with a 30 -g bending force and 10-g/
A

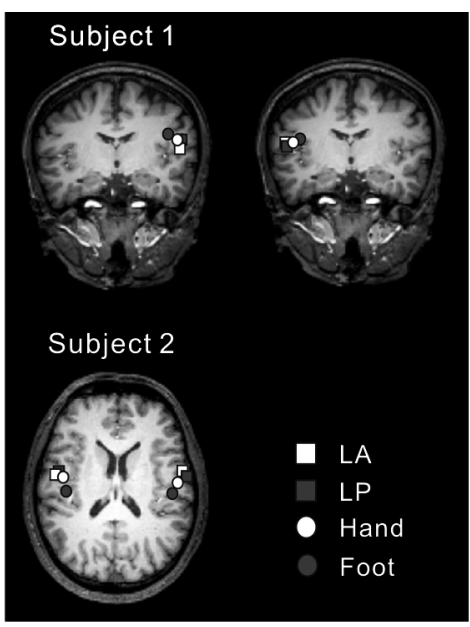

B
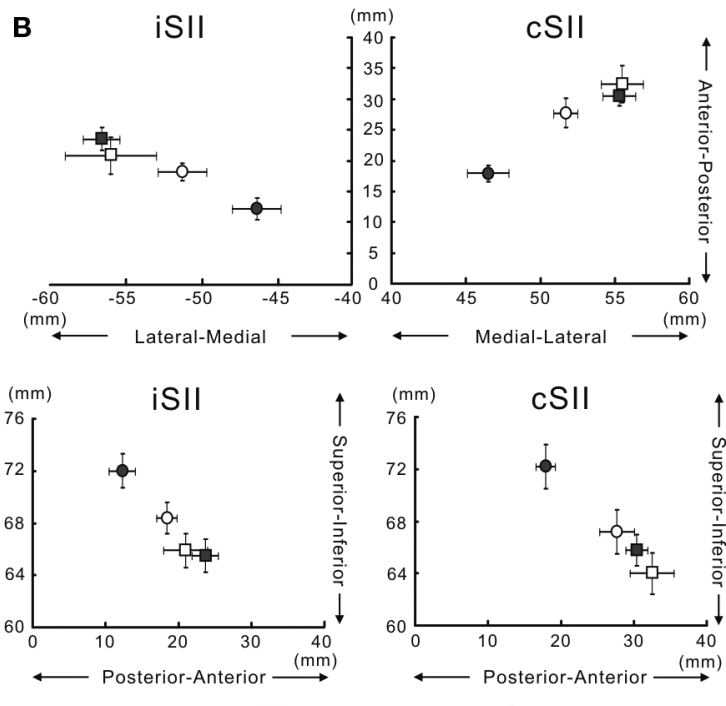

$\square$ LA

LP OHand

Foot

FIGURE 5 | (A) ECD locations for LA, LP, Hand and Foot superimposed on 2D MR images in two representative subjects. Source locations of Subject 1 are superimposed on the coronal plane and those of Subject 2, on the axial plane. The ECDs for bilateral SII responses were located in the upper bank of the Sylvian fissure in the left and right hemispheres. White and gray squares indicate the locations for LA and LP, respectively. White and gray circles indicate the locations for Hand and Foot, respectively. (B) Schematic drawing of spatial relationships of the ECDs for SII among each stimulation point. Upper figures depict the medial-lateral and anterior-posterior directions. Lower figures illustrate the superior-inferior and anterior-posterior directions. LA and LP are located most lateral, anterior and inferior, while the ECD for Foot is located most medial, posterior, and superior. Bars indicate standard error (SE). Adopted from Sakamoto et al. (2008b).

$\mathrm{mm}^{2}$ bending pressure, and compared the cortical activation with toes and fingertips. They showed the cortical activation, which was located on the contralateral postcentral gyrus, and organized medially-to-laterally in the order of toes, fingertips, and tongue tip.

Pardo et al. (1997) stimulated the right or left side of the protruding tongue at a rate of $1 \mathrm{~Hz}$ with a wooden stick, and rCBF was measured using PET. Stimulation of the right side of the tongue produced a contralateral response in SI, while left side stimulation activated bilateral SI. 


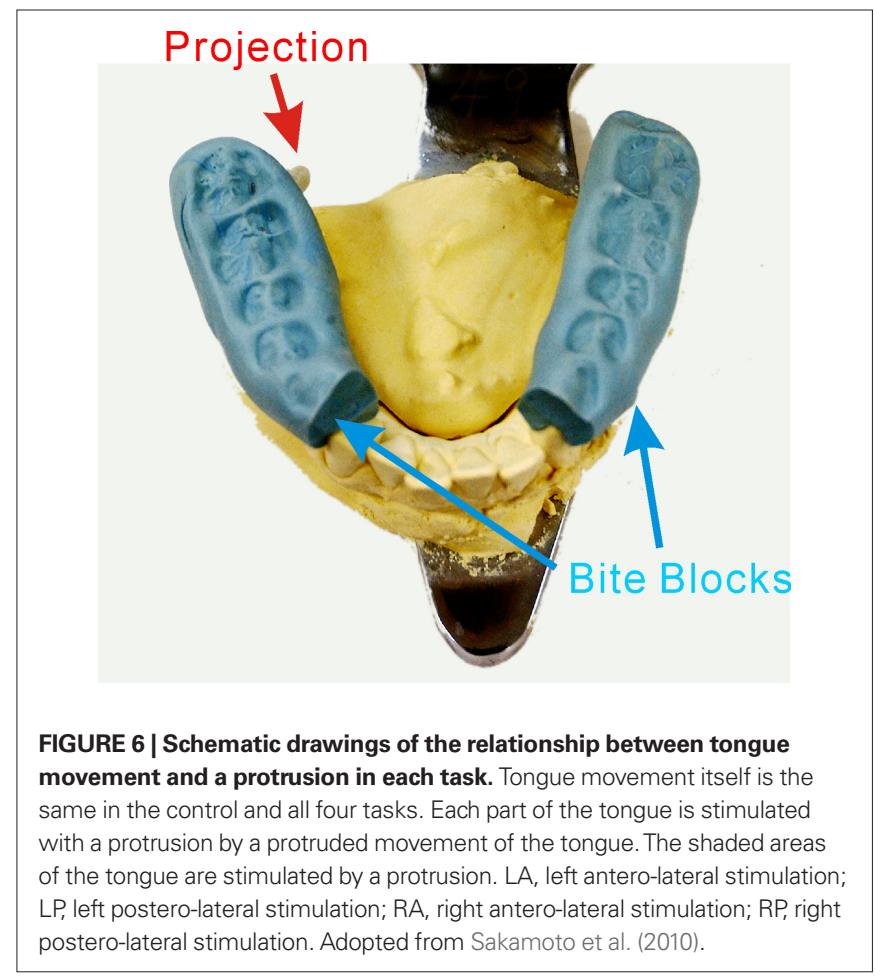

Miyamoto et al. (2006), who used fMRI, made a long stick with a grooved rubber at its tip. The stick used for stimulation was fixed on a table that was set on both edges of the scanner bed to avoid it touching the subject's body. The stick was allowed to rotate around its long axis to minimize the possibility of touching the surrounding structures. The oscillating movement of the stick provided oscillating strokes of $\sim 5 \mathrm{~mm}$ at the contact zone. The anterior part of the tongue, $1 \mathrm{~cm}$ to the right of the midline, was stimulated. Stimulation was provided by the same well-trained experimenter to minimize the variability of stimuli across the subjects. They identified the somatotopic representation of the lips, teeth and tongue in SI, and examined the rostro-caudal changes in the somatotopic organization in SI in terms of the overlap between each sensory representation. In the rostral portion of the postcentral gyrus, the representation of teeth was located significantly superior to that of the tongue and inferior to that of the lip, consistent with the classical "sensory homunculus" proposed by Penfield, while this somatotopic representation became unclear in the middle and caudal portions of postcentral gyrus. The overlap between each representation in the middle and caudal portions of the postcentral gyrus was significantly greater than that in the rostral portion of the postcentral gyrus.

Minato et al. (2009) also used fMRI, and delivered somatosensory stimulation to either side of the tongue at a constant frequency of $2 \mathrm{~Hz}$ with acrylic balls (diameter: $8 \mathrm{~mm}$ ) attached to the ends of two plastic sticks (inner cylinders) that were incorporated in two plastic tubes (outer cylinders) stabilized at the occlusal surface of the bilateral posterior regions of the splint. The proximal end of the $100-\mathrm{cm}$ extension was attached to the inner cylinders of the mandibular splint, while the distal end was equipped with a stopper at $15 \mathrm{~mm}$, which allowed constant displacement of the acrylic balls against the posterior edge of the tongue on each side. With this set-up, a well-trained experimenter could stimulate a specific targeted region without touching surrounding structures. They investigated whether the pattern of hemispheric cortical activation by tactile tongue stimulation differed, with special attention to the preferred chewing side. As the results, the number of activated voxels in S1 contralateral to the preferred chewing side was significantly greater than that in S1 contralateral to the non-preferred chewing side.

In our MRI study, we compared the brain activities following stimulation of the postero-lateral part of the tongue with those following stimulation of the antero-lateral part (Sakamoto et al., 2010). To stimulate different areas of the tongue, we fabricated an intraoral device, which was the same as used in MEG studies (Sakamoto et al., 2008a,b). In addition, the jaws of the subject were positioned based on centric occlusion and opened about $5 \mathrm{~mm}$ between the upper and lower teeth to make a small space that was important to build the projection for stimulating the tongue. These blocks were used to create a wide space from the right to left canine teeth to allow comfortable frontal movement of the tongue. Then, we made four grooves on the lingual side of this device, which were positioned on the lingual cusp of the first premolar of the lower jaw and the distal-lingual cusp of the second molar of the lower jaw bilaterally. Next, we made a projection with polymethylmethacrylate on each groove. The projection has an elliptical shape and is $3 \mathrm{~mm}$ in diameter and $3 \mathrm{~mm}$ in height. This projection is easily attached and detached, but very stable during experiments.

As a control task, the subjects were required to perform the tongue-protruding movement while no projection was set on the device. In the other four tasks, the subjects performed the movement with a projection. The projection was set at four positions to stimulate specific areas of the tongue: the left antero-lateral, left postero-lateral, right antero-lateral, and right postero-lateral areas (Figure 6). In each task, only one projection was set for the target area. After each session, this projection was replaced with another groove; which was attached and detached by the operator out of the MR gantry. To investigate only the somatosensoryrelated activation by removing the motor-related activation and somatosensory-related activation for the device and/or intraoral structures, we analyzed subtraction images obtained from the contrasts as follows: the task in which the projection was on the left antero-lateral of the tongue (left antero-lateral) minus Nonprojection task (Control) (LA), left postero-lateral minus Control (LP), right antero-lateral minus Control (RA), and right posterolateral minus Control (RP).

Stimulation of the left and right postero-lateral parts of the tongue induced significant activity in the SI and Brodmann area 40 (BA 40) in the right hemisphere and the anterior cingulate cortex (ACC) (Figure 7). In contrast, antero-lateral stimulation produced activity only in the right SI (Figure 8). The activated region in SI was significantly larger following stimulation of the posterior than anterior part. These results indicate that a clear difference exists in somatosensory processing between stimulation of the antero-lateral and postero-lateral parts of the tongue, and the right hemisphere is dominant for the stimulation of both antero-lateral and posterolateral areas. As anatomical data in humans, the anterior two-thirds of the tongue are innervated by the afferent fibers that travel in a branch of the trigeminal nerve $(\mathrm{V})$ called the lingual nerve. The 


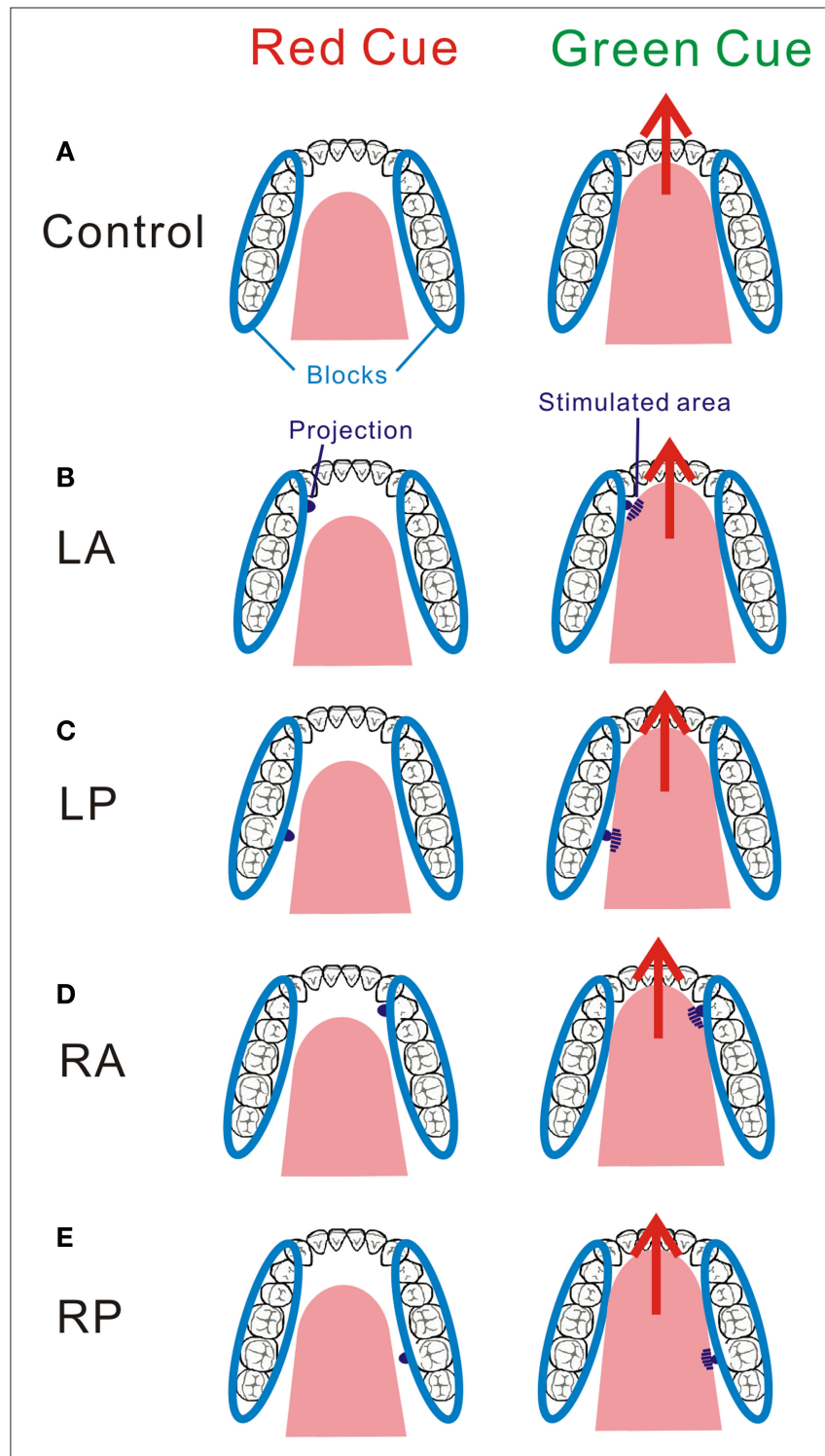

FIGURE 7 | Group activation map showing activated brain regions in four conditions. The figures around SI areas were enlarged for all conditions. Using the Statistical Parametric Mapping 2 (SPM2, Wellcome Department of Cognitive Neurology, London, UK) template, areas showing an increase in BOLD-signal are superimposed on a 3D-rendered standard brain. Although our statistical threshold was $P<0.001$ (uncorrected), the threshold was lowered to $P<0.05$ (uncorrected) for this figure for display purposes. Adopted from Sakamoto et al. (2010).

posterior one-third of the tongue is innervated by the afferent fibers that travel in the lingual branch of the glossopharyngeal nerve (IX) (Kandel et al., 1991). From the results of our study, therefore, the antero-lateral part of the tongue should be innervated by the trigeminal nerve, but the postero-lateral part may be innervated by both the trigeminal and glossopharyngeal nerves, which may underlie the clear differences in brain activities, although the border of the innervation zone and pattern between the trigeminal nerve and the glossopharyngeal nerve is vague and unclear anatomically. Fitzgerald and Law (1958) reported a connection between the lingual nerve and hypoglossal nerve in the tongue. In their

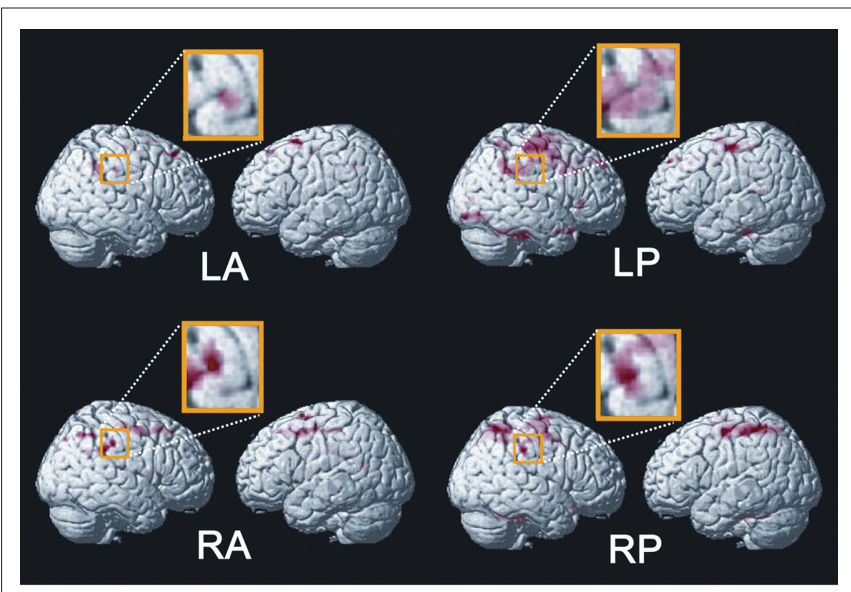

FIGURE 8 | Neural activation in the anterior cingulate cortex (ACC) overlaid on an anatomically normalized MRI. Only LP and RP stimulation produced these activities. A brighter color represents a higher statistical significance. The threshold is $P<0.001$ (uncorrected) in LP and $P<0.005$ (uncorrected), respectively. Adopted from Sakamoto et al. (2010).

study, the peripheral parts of the lingual and hypoglossal nerves were dissected in forty human specimens obtained from 25 adult subjects. We speculate that the same connection is formed between the lingual nerve and glossopharyngeal nerve. Doty et al. (2009) also suggested that branches of the glossopharyngeal nerve extend anteriorly beyond the sulcus terminalis and circumvallate papillae, with extensions occurring along the lateral lingual margin anterior to the foliate papillae. In addition, anastomoses were identified between the glossopharyngeal nerve and the lingual nerve, raising the possibility of functional interactions between the trigeminal nerve and the glossopharyngeal nerve. This notion was supported by a recent anatomical study (Zur et al., 2004). Based on these findings, we inferred that the activated region in SI was larger following stimulation of the posterior than anterior part.

In addition to SI, BA 40 immediately lateral to SI was activated during the postero-lateral stimulation. We assumed that the activation of BA 40 included neuronal activation associated with visceral sensation following the stimulation of the posterior tongue. Previous studies demonstrated that the cortical representation of visceral organs differs from that of the somatosensory system, using fMRI (Aziz et al., 2000; Hobday et al., 2001; Lotze et al., 2001; Strigo et al., 2003; Eickhoff et al., 2006; Ladabaum et al., 2007), and MEG (Schnitzler et al., 1999). For instance, Hobday et al. (2001) observed activation in the inferior part of SI and BA 40 following visceral rectal stimulation, but only in SI following somatic anal stimulation. Ladabaum et al. (2007), who recorded brain activity following gastric distension, found no evidence of activation of S1, but found activation in a broad region of BA 40. Taking these studies into consideration, it is likely that BA 40 was activated following visceral stimulation, but more studies will be necessary to determine the relationship between the neural activity of BA 40 and visceral sensation and to clarify the characteristics of this area with respect to tongue stimulation.

In general, the ACC plays an important role in sensory, motor, cognitive, and emotional information (Bush et al., 2000) and pain processing (Schnitzler and Ploner, 2000; Vogt, 2005; Qiu et al., 
2006). Our results demonstrated that the ACC was activated only during the postero-lateral stimulation. Some studies showed the ACC to often be concerned with visceral sensation. For example, Hobday et al. (2001) noted that the ACC was activated by visceral stimulation, not by somatic stimulation, and it appears that their results are consistent with our findings. Thus, we considered that our ACC activation reflected the attributions of the viscera, because the viscera have a complex peripheral nervous system that allows for a wide variety of autonomic functions (Ness and Gebhart, 1990).

Our fMRI results showed a cortical representation in the right hemisphere, but not left hemisphere. By contrast, in our MEG studies (Sakamoto et al., 2008a,b) and neuroimaging studies (Pardo et al., 1997; Minato et al., 2009), bilateral activations were observed, not showing a right dominant response. There are three possible explanations for the discrepancy between our neuroimaging findings and some previous studies including our own MEG studies. The first possibility is that somatosensory processing includes asymmetric neural activation. That is, as several neuroimaging studies already showed (Perlmutter et al., 1987; Fox and Applegate, 1988; Naito et al., 2005; Nihashi et al., 2005; Eickhoff et al., 2008), the brain's response should be stronger in the right hemisphere than the left for somatosensory processing. We believe that the present study also indicated this asymmetric neural activation. Indeed, our method of stimulation may be unable to elicit clear activation in the left hemisphere, compared to general electrical stimulation. If so, it might be difficult to detect the response in the left hemisphere. The second possibility is a negative motor effect on the left somatosensory areas. Some neuroimaging studies have also provided evidence that activation of the sensorimotor cortex representing the oral and facial regions during volitional swallowing and mastication showed left hemispheric preference (Martin et al., 2004, 2007; Shinagawa et al., 2004). From these studies, there is a possibility that active movement of the tongue affects SI activity in the left hemisphere. Indeed, many studies have investigated somatosensory-motor integration by recording SEPs during voluntary movement. Characteristically, the amplitudes of short-latency components are attenuated, while those of longlatency are enhanced (Giblin, 1964; Kakigi, 1986; Hoshiyama and Sheean, 1998; Rossini et al., 1999; Valeriani et al., 2001; Nakata et al.,

\section{REFERENCES}

Altenmüller, E., Cornelius, C. P., and Buettner, U. W. (1990). Somatosensory evoked potentials following tongue stimulation in normal subjects and patients with lesions of the afferent trigeminal system. Electroencephalogr. Clin. Neurophysiol. 77, 403-415.

Aziz, Q., Thompson, D. G., Ng, V. W., Hamdy, S., Sarkar, S., Brammer, M. J., Bullmore, E. T., Hobson, A., Tracey, I., Gregory, L., Simmons, A., and Williams, S. C. (2000). Cortical processing of human somatic and visceral sensation. J. Neurosci. 20, 2657-2663.

Bessho, H., Shibukawa, Y., Shintani, M., Yajima, Y., Suzuki, T., and Shibahara, T. (2007). Localization of palatal area in
2003), and this phenomenon is termed "gating". This gating effect has been also researched by recording SEFs, and similar results were found regarding the cortical responses. That is, the early responses generated from SI were attenuated during voluntary movement, whereas the late responses in SII were strengthened (Rossini et al., 1989; Kakigi et al., 1995, 1997; Huttunen et al., 1996; Forss and Jousmäki, 1998; Lin et al., 2000). Such modulation also occurred in an fMRI study (Hinkley et al., 2007). A third explanation is that the above two possibilities may be interrelated.

\section{CONCLUSION}

The present study showed the human brain's response after stimulation of the tongue. Because of technical difficulty in stimulating the tongue, researchers have had to make special devices to clarify the neural mechanisms for somatosensory processing. In the somatotopic representation of SI, the face and oral regions as well as the hand area occupied larger regions (Penfield and Boldrey, 1937), indicating an important role in the human somatosensory system.

Future studies must resolve several issues pertaining to somatosensory processing of the tongue. First, extant studies have focused mainly on the earliest component of SEPs/SEFs, generated from area $3 \mathrm{~b}$ in SI. They did not analyze other components, which were recorded on stimulation of the hand, such as the P25, N35, P45, N60, and frontal N30 (Nakata et al., 2003; Kida et al., 2004). These components should be analyzed. Second, the characteristics of the tongue SII should be clarified because few studies have investigated the neural mechanisms involved (see Responses from Secondary Somatosensory Cortex). Third, the devices for stimulating the tongue have depended on individual researchers. Therefore, standard methods need to be established.

We believe that our findings provide valuable information on the neural mechanisms relating to the oral and maxillofacial regions.

\section{ACKNOWLEDGMENT}

This article was supported by grants from the Japan Society for the Promotion of Science for Young Scientists to Kiwako Sakamoto. organization of the lateral sulcus of owl monkeys: area 3b, S-II, and a ventral somatosensory area. J. Comp. Neurol. 282, 169-190.

Decety, J., Perani, D., Jeannerod, M., Bettinardi, V., Tadary, B., Woods, R., Mazziotta, J. C., and Fazio, F. (1994). Mapping motor representations with positron emission tomography. Nature 371, 600-602.

Del Gratta, C., Della Penna, S., Ferretti, A., Franciotti, R., Pizzella, V., Tartaro, A. Torquati, K., Bonomo, L., Romani, G.L., and Rossini, P.M. (2002). Topographic organization of the human primary and secondary somatosensory cortices: comparison of $\mathrm{AMRI}$ and MEG findings. Neuroimage 17, 1373-1383.

Del Gratta, C., Della Penna, S., Tartaro, A., Ferretti,A., Franciotti, R., Torquati,
K., Bonomo, L., Romani, G. L., and Rossini, P. M. (2000). Topographic organization of the human primary and secondary somatosensory areas: an fMRI study. Neuroreport 11, 2035-2043.

Disbrow, E., Roberts, T., and Krubitzer, L. (2000). Somatotopic organization of cortical fields in the lateral sulcus of Homo sapiens: evidence for SII and PV. J. Comp. Neurol. 418, 1-21.

Disbrow, E. A., Hinkley, L. B., and Roberts, T. P. (2003). Ipsilateral representation of oral structures in human anterior parietal somatosensory cortex and integration of inputs across the midline. J. Comp. Neurol. 467, 487-495.

Doty, R. L., Cummins, D. M., Shibanova, A., Sanders, I., and Mu L. (2009). Lingual distribution of the human 
glossopharyngeal nerve. Acta Otolaryngol. 129, 52-56.

Eickhoff, S. B., Grefkes, C., Fink, G. R., and Zilles, K. (2008). Functional lateralization of face, hand, and trunk representation in anatomically defined human somatosensory areas. Cereb. Cortex 18 , 2820-2830.

Eickhoff, S. B., Lotze, M., Wietek, B., Amunts, K., Enck, P., and Zilles, K. (2006). Segregation of visceral and somatosensory afferents: an fMRI and cytoarchitectonic mapping study. Neuroimage 31, 1004-1014.

Fitzgerald, M. J., and Law, M. E. (1958). The peripheral connexions between the lingual and hypoglossal nerves. $J$. Anat. 92, 178-188.

Forss, N., and Jousmäki, V. (1998). Sensorimotor integration in human primary and secondary somatosensory cortices. Brain Res. 781, 259-267.

Fox, P. T., and Applegate, C. N. (1988). Right-hemisphere dominance for somatosensory processing in humans. Soc. Neurosci. Abstr. 18, 760.

Garcha, H. S., and Ettlinger, G. (1978). The effects of unilateral or bilateral removals of the second somatosensory cortex (area SII): a profound tactile disorder in monkeys. Cortex 14,319-326.

Giblin, D. R. (1964). Somatosensory evoked potentials in healthy subjects and in patients with lesions of the nervous system. Ann. N. Y. Acad. Sci. $112,93-142$.

Hari, R., Karhu. J., Hämäläinen. M., Knuutila, J., Salonen, O., Sams, M., and Vilkman, V. (1993). Functional organization of the human first and second somatosensory cortices: a neuromagnetic study. Eur. J. Neurosci. 5, 724-734.

Hari, R., Levänen, S., and Raij, T. (2000). Timing of human cortical functions during cognition: role of MEG. Trends Cogn. Sci. 4, 455-462.

Hinkley, L. B., Krubitzer, L. A., Nagarajan, S. S., and Disbrow, E. A. (2007). Sensorimotor integration in S2, PV, and parietal rostroventral areas of the human Sylvian fissure. J. Neurophysiol. 97, 1288-1297.

Hobday, D. I., Aziz, Q., Thacker, N., Hollander, I., Jackson, A., and Thompson, D. G. (2001). A study of the cortical processing of ano-rectal sensation using functional MRI. Brain $124,361-368$.

Hoshiyama, M., and Sheean, G. (1998). Changes of somatosensory evoked potentials preceding rapid voluntary movement in Go/No-go choice reaction time task. Brain Res. Cogn. Brain Res. 7, 137-142.

Huttunen, J., Komssi, S., and Lauronen, L. (2006). Spatial dynamics of population activities at $\mathrm{S} 1$ after median and ulnar nerve stimulation revisited: an MEG study. Neuroimage 32, 1024-1031.

Huttunen, J., Wikström, H., Korvenoja, A., Seppäläinen, A. M., Aronen, H., and Ilmoniemi, R. J. (1996). Significance of the second somatosensory cortex in sensorimotor integration: enhancement of sensory responses during finger movements. Neuroreport 7, 1009-1012.

Inui, K., Wang, X., Tamura, Y., Kaneoke, Y., and Kakigi, R. (2004). Serial processing in the human somatosensory system. Cereb. Cortex 14, 851-857.

Ishiko, N., Hanamori, T., and Murayama, N. (1980). Spatial distribution of somatosensory responses evoked by tapping the tongue and finger in man. Electroencephalogr. Clin. Neurophysiol. 50, 1-10.

Iwamura, Y. (1998). Hierarchical somatosensory processing. Curr. Opin. Neurobiol. 8, 522-528.

Jain, N., Qi, H., Catania, K. C., and Kaas, J. H. (2001). Anatomic correlates of the face and oral cavity representations in the somatosensory cortical area $3 \mathrm{~b}$ of monkeys. J. Comp. Neurol. $429,455-468$.

Jasper, H. H. (1958). The ten-twenty electrode system of the International Federation. Electroencephalogr. Clin. Neurophysiol. 10, 371-375.

Kakigi, R. (1986). Ipsilateral and contralateral SEP components following median nerve stimulation: effects of interfering stimuli applied to the contralateral hand. Electroencephalogr. Clin. Neurophysiol. 64, 246-259.

Kakigi, R., Hoshiyama, M., Shimojo, M., Naka, D., Yamasaki, H., Watanabe, S., Xiang, J., Maeda, K., Lam, K., Itomi, K., and Nakamura, A. (2000). The somatosensory evoked magnetic fields. Prog. Neurobiol. 61, 495-523.

Kakigi, R., Koyama, S., Hoshiyama, M., Watanabe, S., Shimojo, M., and Kitamura, Y. (1995). Gating of somatosensory evoked responses during active finger movements magnetoencephalographic studies. J. Neurol. Sci. 128, 195-204.

Kakigi, R., Shimojo, M., Hoshiyama, M., Koyama, S., Watanabe, S., Naka, D., Suzuki, H., and Nakamura, A. (1997). Effects of movement and movement imagery on somatosensory evoked magnetic fields following posterior tibial nerve stimulation. Brain Res. Cogn. Brain Res. 5, 241-253.

Kandel, E., Schwartz, J. H., and Jessell, T. M. (1991).Principles of Neural Science, 3rd Edn. Norwalk, CT: Appleton and Lange, 519.

Karhu, J., Hari, R., Lu, S. T., Paetau, R., and Rif, J. (1991). Cerebral magnetic fields to lingual stimulation. Electroencephalogr. Clin. Neurophysiol. $80,459-468$.
Kida, T., Nishihira, Y., Wasaka, T., Sakajiri, Y., and Tazoe, T. (2004). Differential modulation of the short- and longlatency somatosensory evoked potentials in a forewarned reaction time task. Clin. Neurophysiol. 115 2223-2230.

Korvenoja, A., Wikström, H., Huttunen, J., Virtanan, J., Laine, P., Aronen, H. J., Seppäläinen A. M., and Ilmoniemi, R. J. (1995). Activation of ipsilateral primary sensorimotor cortex by median nerve stimulation. Neuroreport 6 , 2589-2593.

Krubitzer, L. A., Sesma, M. A., and Kaas, J. H. (1986). Microelectrode maps, myeloarchitecture, and cortical connections of three somatotopically organized representations of the body surface in the parietal cortex of squirrels. J. Comp. Neurol. 250, 403-430.

Ladabaum, U., Roberts, T. P., and McGonigle, D. J. (2007). Gastric fundic distension activates fronto-limbic structures but not primary somatosensory cortex: a functional magnetic resonance imaging study. Neuroimage 34, 724-732.

Lin, Y. Y., Simões, C., Forss, N., and Hari, R. (2000). Differential effects of muscle contraction from various body parts on neuromagnetic somatosensory responses. Neuroimage 11, 334-340.

Logothetis, N. K., Pauls, J., Augath, M., Trinath, T., and Oeltermann, A. (2001). Neurophysiological investigation of the basis of the fMRI signal. Nature 412, 150-157.

Lotze, M., Wietel, B., Birbaumer, N., Ehrhardt, J., Grodd, W., and Enck, P. (2001).Cerebral activation during anal and rectal stimulation. Neuroimage 14 1027-1034.

Maeda, K., Kakigi, R., Hoshiyama, M., and Koyama, S. (1999). Topography of the secondary somatosensory cortex in humans: a magnetoencephalo-graphic study. Neuroreport 10, 301-306.

Maezawa, H., Yoshida, K., Nagamine, T., Matsubayashi, J., Enatsu, R., Bessho, K., and Fukuyama, H. (2008). Somatosensory evoked magnetic fields following electric tongue stimulation using pin electrodes. Neurosci. Res. 62 , 131-139.

Maloney, S.R., Bell, W.L.,Shoaf, S.C., Blair, D., Bastings, E. P., Good, D. C., and Quinlivan, L. (2000). Measurement of lingual and palatine somatosensory evoked potentials. Clin. Neurophysiol. 111, 291-296.

Manger, P. R., Woods, T. M., and Jones, E. G. (1995). Representation of the face and intraoral structures in area $3 \mathrm{~b}$ of the squirrel monkey (Saimiri sciureus) somatosensory cortex, with special reference to the ipsilateral representation. J. Comp. Neurol. 362, 597-607.
Manger, P. R., Woods, T. M., and Jones, E. G. (1996). Representation of face and intra-oral structures in area $3 \mathrm{~b}$ of macaque monkey somatosensory cortex. J. Comp. Neurol. 371, 513-521.

Martin, R., Barr, A., MacIntosh, B., Smith, R., Stevens, T., Taves, D., Gati, J., Menon, R., and Hachinski, V. (2007). Cerebral cortical processing of swallowing in older adults. Exp. Brain Res. 176, 12-22.

Martin, R. E., MacIntosh, B. J., Smith, R. C., Barr, A. M., Stevens, T. K., Gati, J. S., and Menon, R. S. (2004). Cerebral areas processing swallowing and tongue movement are overlapping but distinct: a functional magnetic resonance imaging study. J. Neurophysiol. 92, 2428-2443.

Mauguière, F., Merlet, I., Forss, N., Vanni, S., Jousmäki, V., Adeleine, P., and Hari, R. (1997). Activation of a distributed somatosensory cortical network in the human brain: a dipole modelling study of magnetic fields evoked by median nerve stimulation. Part II: effects of stimulus rate, attention and stimulus detection. Electroencephalogr. Clin. Neurophysiol. 104, 290-295.

Mima, T., Nagamine, T., Nakamura, K., and Shibasaki, H. (1998). Attention modulates both primary and second somatosensory cortical activities in humans: a magnetoencephalographic study. J. Neurophysiol. 80, 2215-2221.

Minato,A., Ono, T., Miyamoto, J.J.,Honda, E., Kurabayashi, T., and Moriyama, K. (2009). Preferred chewing sidedependent two-point discrimination and cortical activation pattern of tactile tongue sensation. Behav. Brain Res. 203, 118-126.

Miyamoto, J. J., Honda, M., Saito, D. N., Okada, T., Ono, T., Ohyama, K., and Sadato, N. (2006). The representation of the human oral area in the somatosensory cortex: a functional MRI Study. Cereb. Cortex 16, 669-675.

Naito,E., Roland,P.E., Grefkes, C., Choi, H. J., Eickhoff, S., Geyer, S., Zilles, K., and Ehrsson, H. H. (2005). Dominance of the right hemisphere and role of area 2 in human kinesthesia.J. Neurophysiol. 93, 1020-1034.

Nakahara, H., Nakasato, N., Kanno, A., Murayama, S., Hatanaka, K. Itoh, H., and Yoshimoto, T. (2004). Somatosensory-evoked fields for gingiva, lip, and tongue. J. Dent. Res. 83, 307-311.

Nakamura, A., Yamada, T., Goto, A., Kato, T., Ito, K., Abe, Y., Kachi, T., and Kakigi, R. (1998). Somatosensory homunculus as drawn by MEG. Neuroimage 7, 377-386.

Nakata, H., Inui, K., Wasaka, T., Nishihira, Y., and Kakigi, R. (2003). Mechanisms of differences in gating effects on short-and long-latency somatosensory 
evoked potentials relating to movement. Brain Topogr. 15, 211-222.

Nakata, H., Inui, K., Wasaka, T., Tamura, Y., Tran, T. D., Qiu, Y., Wang, X., Nguyen, T. B., and Kakigi, R. (2004). Movements modulate cortical activities evoked by noxious stimulation. Pain 107, 91-98.

Ness, T. J., and Gebhart, G. F. (1990). Visceral pain: a review of experiment studies. Pain 41, 167-234.

Nguyen, B. T., Inui, K., Hoshiyama, M., Nakata, H., and Kakigi, R. (2005). Face representation in the human secondary somatosensory cortex. Clin. Neurophysiol. 116, 1247-1253.

Nihashi, T., Naganawa, S., Sato, C., Kawai, H., Nakamura, T., Fukatsu, H., Ishigaki, T., and Aoki, I. (2005). Contralateral and ipsilateral responses in primary somatosensory cortex following electrical median nerve stimulation - an fMRI study. Clin. Neurophysiol. 116, 842-848.

Pardo, J. V., Wood, T. D., Costello, P. A., Pardo, P. J., and Lee, J. T. (1997). PET study of the localization and laterality of lingual somatosensory processing in humans. Neurosci. Lett. 234, 23-26.

Penfield, W., and Boldrey, E. (1937). Somatic motor and sensory representation in the cerebral cortex of man as studied by electrical stimulation. Brain 60, 389-443.

Perlmutter, J.S., Powers, W.J., Herscovitch, P., Fox, P. T., and Raichle, M. E. (1987). Regional asymmetries of cerebral blood flow, blood volume, and oxygen utilization and extraction in normal subjects. J. Cereb. Blood Flow Metab. 7, 64-67.

Qiu, Y., Noguchi, Y., Honda, M., Nakata, H., Tamura, Y., Tanaka, S., Sadato, N., Wang, X., Inui, K., and Kakigi, R. (2006). Brain processing of the signals ascending through unmyelinated $\mathrm{C}$ fibers in humans: an eventrelated functional magnetic resonance imaging study. Cereb. Cortex 16, 1289-1295.

Ridley, R. M., and Ettlinger, G. (1976). Impaired tactile learning and retention after removals of the second somatic sensory projection cortex (SII) in the monkey. Brain Res. 109, 656-660.

Ridley, R. M., and Ettlinger, G. (1978). Further evidence of impaired tactile learning after removals of the second somatic sensory projection cortex (SII) in the monkey. Exp. Brain Res. 31, 475-488.

Romo, R., Hernandez, A., Zainos, A., Lemus, L., and Brody, C. D. (2002). Neuronal correlates of decisionmaking in secondary somatosensory cortex. Nat. Neurosci. 5, 1217-1225.

Rossini, P. M., Babiloni, C., Babiloni, F., Ambrosini, A., Onorati, P., Carducci, F., and Urbano, A. (1999). "Gating" of human short-latency somatosensory evoked cortical responses during execution of movement. A high resolution electroencephalography study. Brain Res. 843, 161-170.

Rossini, P. M., Narici, L., Romani, G. L. Peresson, M., Torrioli, G., and Traversa, R. (1989). Simultaneous motor output and sensory input: cortical interference site resolved in humans via neuromagnetic measurements. Neurosci. Lett. 96, 300-305.

Ruben, J., Schwiemann, J., Deuchert, M., Meyer, R., Krause, T., Curio, G., Vilringer, K., Kurth, R., and Villringer, A. (2001). Somatotopic organization of human secondary somatosensory cortex. Cereb. Cortex 11, 463-473.

Sakai, K., Watanabe, E., Onodera, Y., Itagaki, H., Yamamoto, E., Koizumi, H., and Miyashita, Y. (1995). Functional mapping of the human somatosensory cortex with echo-planar MRI. Magn. Reson. Med. 33, 736-743.

Sakamoto, K., Nakata, H., Inui, K., Perrucci, M. G., Del Gratta1, C., Kakigi, R., and Romani, G. L. (2010). A difference exists in somatosensory processing between the anterior and posterior parts of the tongue. Neurosci. Res. 66, 173-179.

Sakamoto, K., Nakata, H., and Kakigi, R. (2008a). Somatosensory evoked magnetic fields following stimulation of the tongue in humans. Clin. Neurophysiol. 119, 1664-1673.

Sakamoto, K., Nakata, H., and Kakigi, R. (2008b). Somatotopic representation of the tongue in human secondary somatosensory cortex. Clin. Neurophysiol. 119, 2125-2134.

Schnitzler, A., and Ploner, M. (2000). Neurophysiology and functional neuroanatomy of pain perception. J. Clin. Neurophysiol. 17, 592-603.

Schnitzler, A., Volkmann, J., Enck, P., Frieling, T., Witte, O. W., and Freund, H. J. (1999). Different cortical organization of visceral and somatic sensation in humans. Eur. J. Neurosci. 11 305-315.

Shinagawa, H., Ono, T., Honda, E., Sasaki, T., Taira, M., Iriki, A., Kuroda, T., and Ohyama, K. (2004). Chewing-side preference is involved in differential cortical activation patterns during tongue movements after bilatera gum-chewing: a functional magnetic resonance imaging study. J. Dent. Res. $83,762-766$.

Steinmetz, P. N., Roy, A., Fitzgerald, P. J., Hsiao, S. S., Johnson, K. O., and Niebur, E. (2000). Attention modulates synchronized neuronal firing in primate somatosensory cortex. Nature 444, 187-190.

Strigo, I.A., Duncan, G.H., Boivin, M., and Bushnell, M.C. (2003). Differentiation of visceral and cutaneous pain in the human brain. J. Neurophysiol. 89 , 3294-3303.

Tamura, Y., Shibukawa, Y., Shintani, M., Kaneko, Y., and Ichinohe, T. (2008). Oral structure representation in human somatosensory cortex. Neuroimage 43, 128-135.

Torquati, K., Pizzella, V., Della Penna, S. Franciotti, R., Babiloni, C., Romani, G. L., and Rossini, P. M. (2003). 'Gating' effects of simultaneous peripheral electrical stimulations on human secondary somatosensory cortex: a whole-head MEG study. Neuroimage 20, 1704-1713.

Valeriani, M., Insola, A., Restuccia, D., Le Pera, D., Mazzone, P., Altibrandi, M. G. and Tonali, P. (2001). Source generators of the early somatosensory evoked potentials to tibial nerve stimulation: an intracerebral and scalp recording study. Clin. Neurophysiol. 112, 1999-2006.

Vogt, B.A. (2005). Pain and emotion interactions in subregions of the cingulate gyrus. Nat. Rev. Neurosci. 6, 533-544.

Wasaka, T., Hoshiyama, M., Nakata, H., Nishihira, Y., and Kakigi R. (2003). Gating of somatosensory evoked magnetic fields during the preparatory period of self-initiated finger movement. Neuroimage 20, 1830-1838.

Wasaka, T., Nakata, H., Akatsuka, K., Kida, T., Inui, K., and Kakigi R. (2005). Differential modulation in human primary and secondary somatosensory cortices during the preparatory period of self-initiated finger movement. Eur. J. Neurosci. 22, 1239-1247.

Zur, K. M., Mu, L., and Sanders, I. (2004). Distribution pattern of the human lingual nerve. Clin. Anat. 17, 88-92.

Conflict of Interest Statement: The authors declare that the research was conducted in the absence of any commercial or financial relationships that could be construed as a potential conflict of interest.

Received: 15 July 2010; paper pending published: 13 August 2010; accepted: 12 September 2010; published online: 01 November 2010.

Citation: Sakamoto K, Nakata H, Yumoto $M$ and Kakigi $R$ (2010) Somatosensory processing of the tongue in humans. Front. Physio. 1:136. doi: 10.3389/ fphys.2010.00136

This article was submitted to Frontiers in Integrative Physiology, a specialty of Frontiers in Physiology.

Copyright (c) 2010 Sakamoto, Nakata, Yumoto and Kakigi. This is an open-access article subject to an exclusive license agreement between the authors and the Frontiers Research Foundation, which permits unrestricted use, distribution, and reproduction in any medium, provided the original authors and source are credited. 